\title{
Criação de Aplicativo Móvel para uso na Assistência de Enfermagem Oncológica: Uma Estratégia de Educação Permanente em Saúde
}

\author{
Creation of Mobile Application for use in Oncology Nursing Assistance: A Strategy for Permanent \\ Health Education \\ Creación de una aplicación móvil para su uso en asistencia de enfermería oncológica: una estrategia \\ para la educación permanente en salud
}

Raphael Braz de Carvalho ${ }^{1 *}$, Magda de Souza Chagas², Ana Lúcia Abrahão da Silva ${ }^{3}$

Como citar esse artigo. de Carvalho, RB; Chagas, MS; da Silva, ALA. Criação de Aplicativo Móvel para uso na Assistência de Enfermagem Oncológica: Uma Estratégia de Educação Permanente em Saúde. Revista Pró-UniverSUS. 2020 Jul./Dez.; 11 (2): 189-191.

\begin{abstract}
Resumo
Introdução: As Tecnologias da Informação e Comunicação (TICs) são um conjunto de tecnologias usadas mundialmente para acesso à informação por profissionais ou qualquer usuário de um sistema que queira mudar sua relação de ensino-aprendizado. Objetivo: Construir um aplicativo móvel voltado para o ensino, ferramenta acadêmica e como apoio na assistência de enfermagem oncológica. Metodologia: Trata-se da Pesquisa Convergente-Assistencial (PCA), uma modalidade de pesquisa qualitativa de caráter direcionado pela imersibilidade, simultaneidade, expansibilidade e dialogicidade que aproxima a pesquisa, assistência e participação dos profissionais envolvidos na assistência de forma ativa no processo de discussão dos problemas, reflexão dos norteadores da prática e construção do conhecimento. A PCA foi delineada na década de 80 e tem foco na inovação das práticas assistenciais da enfermagem. Resultados esperados: Contribuir com profissionais de enfermagem, a partir de aplicativo móvel específico para a assistência de enfermagem oncológica, identificar os conhecimentos necessários para incluir neste aplicativo, possibilitar aquisição e troca de conhecimentos e melhorar o cuidado.
\end{abstract}

Palavras-chave: Cuidados de Enfermagem Oncológica, Aplicativos Móveis, Educação Permanente em Saúde.

\begin{abstract}
Introduction: Information and Communication Technologies (ICTs) are a set of technologies used worldwide to access information by professionals or any user of a system that wants to change their teaching-learning relationship. Objective: To build a mobile application aimed at teaching, an academic tool and as a support in oncology nursing care. Methodology: This is Convergent-Assistance Research (PCA), a qualitative type of research guided by immersibility, simultaneity, expandability and dialogicity that brings together research, assistance and participation of professionals involved in assistance actively in the discussion process problems, reflection of the guiding practice and knowledge construction. The PCA was designed in the 1980 s and focuses on the innovation of nursing care practices. Expected results: Contribute to nursing professionals, using a specific mobile application for oncology nursing care, build, exchange knowledge and improve the safety of patient care.

Keywords: Oncology Nursing Care, Mobile Apps, Permanent Health Education.
\end{abstract}

\section{Resumen}

Introducción: Las Tecnologías de la Información y la Comunicación (TIC) son un conjunto de tecnologías utilizadas a nivel mundial para acceder a la información por parte de profesionales o cualquier usuario de un sistema que quiera cambiar su relación enseñanza-aprendizaje. Objetivo: Construir una aplicación móvil destinada a la docencia, herramienta académica y soporte en la atención de enfermería oncológica. Metodología: Se trata de Investigación Convergente-Asistencial (PCA), una modalidad de investigación cualitativa de carácter dirigida por la inmersión, la simultaneidad, la expansibilidad y la dialogicidad que aglutina la investigación, asistencia y participación de profesionales involucrados en la asistencia activamente en el proceso de discusión. Problemas, reflejo de la práctica rectora y construcción del conocimiento. El PCA fue diseñado en la década de 1980 y se centra en la innovación de las prácticas de atención de enfermería. Resultados esperados: Contribuir a que los profesionales de enfermería, desde una aplicación móvil específica para la asistencia de enfermería oncológica, identifiquen los conocimientos necesarios para incluir en esta aplicación, posibiliten la adquisición e intercambio de conocimientos y mejoren la atención.

Palabras clave: Atención de Enfermería Oncológica; Aplicaciones Móviles; Educación Permanente en Salud.

Afiliação dos autores:

${ }^{1}$ Enfermeiro do Instituto Nacional de Câncer (INCA), Mestrando da Escola de Enfermagem Aurora de Afonso Costa da Universidade Federal Fluminense (UFF). Niterói, RJ. Brasil. ORCID: https://orcid.org/0000-0002-2235-1181

${ }^{2}$ Enfermeira. Doutora em Ciências da Saúde pela Universidade Federal do Rio de Janeiro (UFRJ). Professora Adjunta da Universidade Federal Fluminense (UFF). Programa de Mestrado Profissional em Ensino na Saúde/MPES, UFF, RJ, Brasil. ORCID: https://orcid.org/0000-0002-3616-6745

${ }^{3}$ Enfermeira. Pós Doutora vinculada ao quadro Permanente do Programa de Ciências do Cuidado em Saúde (UFF) e do Mestrado Profissional de Ensino em Saúde (UFF), Professora Titular da Universidade Federal Fluminense, Escola de Enfermagem, RJ, Brasil. ORCID: https://orcid.org/0000-0002-0820-4329

* Email de correspondencia: : phbraz@yahoo.com.br

Recebido em: 07/07/20. Aceito em: 18/10/20. 


\section{Introdução}

O câncer é a segunda principal causa de morte no mundo atingindo principalmente países pobres e em desenvolvimento. $\mathrm{O}$ envelhecimento e os fatores de risco exposto durante a vida aumentam as chances do desenvolvimento desta doença $a^{1,2}$.

$\mathrm{Na}$ mais recente estimativa de casos de câncer para o Brasil, espera-se para o triênio 2020-2022 um total de 625 mil casos novos de câncer ${ }^{3}$. Sendo assim, os profissionais que atuam na Oncologia precisam ter habilidades singulares no conceito, no curso terapêutico e uma compleição psicológica das necessidades dos pacientes com câncer, pois é uma especialidade complexa em todos os seus processos e os profissionais que assistem os doentes com câncer vivenciam situações de luto, esperança e morte, precisam instrumentalizar a assistência de modo que o pesar seja amenizado, mantendo a integralidade do cuidado em oncologia ${ }^{4}$.

O cuidado construído erealizado pelaEnfermagem inclui, na elaboração a perspectiva de compreender o ser humano em seu contexto social, histórico e cultural, não apartando ou deixando de fora estes aspectos na oferta de cuidados gerais prestados à beira do leito ${ }^{5}$. Ao considerarmos o contexto histórico e cultural no processo de aquisição de conhecimento, incorporemos a dinamicidade e a amplitude de ofertas que as pessoas têm vivenciado é também uma necessidade. Assim, buscar e estabelecer novas formas, ofertas e acessos de ensino ao pessoal da enfermagem pode se consolidar, tanto como ofertas e possibilidades de atualização de conhecimento e gratificação pessoal, como também uma medida econômica que no curso do seu desenvolvimento amplia ao mesmo tempo a qualidade da assistência de saúde para usuários/pacientes 6 .

As Tecnologias da Informação e Comunicação (TICs) são um conjunto de tecnologias usadas mundialmente para acesso à informação por profissionais ou qualquer usuário de um sistema que queira mudar sua relação de ensino-aprendizado ${ }^{7}$.

Atualmente encontram-se disponíveis diversos aplicativos móveis educativos voltados para a enfermagem, direcionados tanto para o ensino, quanto para o melhor desenvolvimento do cuidado e assim contribuem para o avanço do cuidado ao paciente ${ }^{8}$. No entanto, poucos são os aplicativos desenvolvidos especificamente para área oncológica, uma área em que a novidade de diagnósticos impõe atualização de técnicas e procedimentos terapêuticos. Em tal contexto, os serviços de saúde, podem se beneficiar cada vez mais do conhecimento e uso de ferramentas tecnológicas de acesso fácil e que apresentem conteúdos voltados para cada especialidade, facilitando a agilidade e usabilidade no trabalho profissional 9 .

$\mathrm{O}$ estudo baseia-se na estratégia de Educação
Permanente em Saúde (EPS) por fomentar o empoderamento e a aprendizagem voltada para o profissional de enfermagem na atuação oncológica como agente ativo diante das necessidades do serviço e dos pacientes com câncer ${ }^{10}$.

\section{Objetivo}

Construir um aplicativo móvel voltado para o ensino, ferramenta acadêmica e apoio na assistência de enfermagem oncológica. Para isso, precisamos identificar os conhecimentos necessários para incluir neste aplicativo, definir a melhor tecnologia para que funcione adequadamente e qual serviço de distribuição de aplicativos se adequa melhor ao produto.

\section{Método}

A metodologia utilizada para a concepção dos dados para elaboração do aplicativo móvel será a convergente-assistencial sendo ela uma modalidade de pesquisa qualitativa que aproxima a pesquisa, assistência e participação dos profissionais envolvidos junto ao processo de discussão dos problemas, reflexão dos norteadores da prática e construção do conhecimento. A PCA sequencia-se por fases para fundamentação teórica e interpretação da síntese dos resultados obtidos por estes profissionais que atuam no cuidado e buscam melhorias para o seu local de trabalho de forma ativa na pesquisa $^{11}$.

Esta abordagem, delineada na década de 80 tem foco na inovação das práticas assistenciais da enfermagem com elementos que dançam pela pesquisa e o cuidado, apresenta rigor no processo de pesquisa, tem seu caráter direcionados pela imersibilidade, simultaneidade, expansibilidade e dialogicidade que assim, fazem a avaliação do fenômeno a partir do potencial de cada profissional envolvido na pesquisa transformando positivamente o local de trabalho ${ }^{12}$.

Os participantes do estudo serão os profissionais de enfermagem cujas atividades estejam envolvidas com os pacientes em tratamento de câncer. O pesquisador irá apresentar a proposta do estudo para os participantes, que após declarar a participação na pesquisa irão assinar o Termo de Consentimento Livre e Esclarecido (TCLE) em duas vias o qual uma via do pesquisador e outra do participante. A proposta de pesquisa foi submetido ao Comitê de Ética e Pesquisa da Universidade Federal Fluminense. Instituto de Saúde de Nova Friburgo. Pólo Universitário de Nova Friburgo da Universidade Federal Fluminense-UFF sob o número 33564820.1.0000.5626. Os dados dos encontros da pesquisa serão coletados por Oficinas de Educação Permanente, anotações, questionários e/ou gravações de voz e retratarão elementos existentes na realidade diária de campo. Para 
análise dos dados será utilizado a transcrição e análise de material acrescentando-se as proposições de maior impacto na construção da pesquisa de conteúdo do aplicativo. $\mathrm{O}$ cenário da pesquisa será uma instituição especializada em tratamento de pacientes com câncer, de atenção terciária localizada na cidade do Rio de Janeiro.

\section{Resultados Esperados}

Espera-se contribuir para os profissionais da enfermagem com um aplicativo móvel relacionado a assistência de enfermagem oncológica que apresente impacto nas ações de saúde pública, e um novo olhar na construção do conhecimento da categoria, com aplicabilidade eficaz no trabalho assistencial por todos os processos do cuidado ao paciente.

\section{Referências}

1. Organização Pan-Americana da Saúde - OPAS Brasil. [homepage na internet]. Folha Informativa - Câncer. [acesso em 10 dez 2019]. Disponível em: https://www.paho.org/bra/index.php?option=com_content\&view=articl e\&id=5588:folha-informativa-cancer\&Itemid=1094"Itemid=1094

2. Organização Mundial da Saúde - OMS 2018. [homepage na internet] Quadro de monitoramento Global da NCD. [acesso em 10 dez 2019]. Disponível em: https://www.who.int/nmh/global_monitoring_framework/ en/

3. Instituto Nacional de Câncer José Alencar Gomes da Silva. Rio de Janeiro: INCA, 2019. [homepage na internet]. Estimativa 2020: incidência de câncer no Brasil. [acesso em 30 mar 2020]. Disponível em: https://www. inca.gov.br/sites/ufu.sti.inca.local/files//media/document//estimativa-2020incidencia-de-cancer-no-brasil.pdf

4. Fabiana Godoys Lins, Sonia Regina de Souza. Formação dos Enfermeiros para o Cuidado em Oncologia. Rev. Enferm. UFPE online [revista em internet] 2018. [acesso em 18 mar 2020]. Disponível em: "https://periodicos.ufpe.br/revistas/revistaenfermagem/article/ view/22652/25857\%20acesso $\% 20 \mathrm{em} \% 2018 / 03 / 2020$ ”

5. Souza, Claudio José de. Produções Tecnológicas dos Mestrados como bases paradigmáticas para a Ciência da Enfermagem. Niterói. Universidade Federal Fluminense, 2018. Tese de Doutorado em Ciências do Cuidado em Saúde. [acesso em 30 mar 2020]. Disponível em: https://app.uff.br/riuff/ handle/1/7242

6. World Health Organization - WHO. [homepage na internet]. Fortalecer a Enfermagem e a Obstetrícia para tornar a Saúde para Todos uma Realidade. [acesso em 18 mar 2020]. Disponível em: https://www.who. int/es/news-room/campaigns/year-of-the-nurse-and-the-midwife-2020/getinvolved/key-messages"of-the-nurse-and-the-midwife-2020/get-involved/ key-messages

7. Teixeira, Elizabeth. Desenvolvivento de Técnologias CuidativoEducacionais. [livro online] Volume 2. Porto Alegre: Moriá, 2020. 398p. Capítulo 5: Métodos e Plataformas Gratuitas para o Desenvolvimento de Aplicativos. [acesso em 12 mar 2020]. Disponível em: file://C:/Users/55219/ Downloads/M\%C3\%A 9 todos $\% 20 \mathrm{e} \% 20$ plataformas $\% 20$ gratuitas $\% 20$ para $\% 20$ desenvolvimento $\% 20 \mathrm{de} \% 20$ aplicativos.pdf

8. Silveira, Maurício de Souza; Cogo, Ana Luísa Petersen. Contribuições das tecnologias educacionais digitais no ensino de habilidades de enfermagem: revisão integrativa. Rev. Gaúcha Enferm., Porto Alegre, 2017. [acesso em 09 mar 2020]. Disponível em: $<\mathrm{http}$ ://www.scielo.br/scielo.php?script $=$ sci_ arttext\&pid=S1983-14472017000200501\&lng=pt\&nrm=iso"nrm=iso>

9. Diniz, Cinthia Martins Menino et al. Contribuições dos aplicativos móveis para a prática do aleitamento materno: revisão integrativa. Acta paul. enferm., São Paulo, Oct. 2019. [acesso em 09 mar 2020]. Disponível em:
$<$ http://www.scielo.br/scielo.php?script=sci_arttext\&pid=S0103-21002019 $000500015 \& \operatorname{lng}=$ en\&nrm=iso"nrm=iso $>$

10. Vicente, Camila et al. Cuidado à pessoa com ferida oncológica: educação permanente em enfermagem mediada por tecnologias educacionais. Rev. Gaúcha Enferm., Porto Alegre, 2019. [acesso em 20 mar 2020]. Disponível em: <http://www.scielo.br/scielo.php?script=sci_arttext. Acesso em 20/03/2020

11. Pivoto, Flávia Lamberti et al. Pesquisa convergente-assistencial: revisão integrativa de produções científicas da enfermagem. Texto contexto - enferm., Florianópolis, Sept. 2013. [acesso em 10 dez 2019]. Disponível em: <http://www.scielo.br/scielo.php?script=sci_arttext\&pid=S0104-07072 013000300034\&lng=en\&nrm=iso"nrm=iso>

12. Trentini, Mercedes; Paim, Lygia; Silva, Denise Maria Guerreiro Vieira da. O método da pesquisa convergente assistencial e sua aplicação na prática de enfermagem. Texto contexto - enferm., Florianópolis, 2017. [acesso em $10 \mathrm{dez}$ 2019]. Disponível em <http://www.scielo.br/scielo. php?script $=$ sci_arttext\&pid $=$ S0104-07072017000400> 